\title{
The stability of a viscous till sheet coupled with ice flow, considered at wavelengths less than the ice thickness
}

\author{
Richard C. A. Hindmarsh \\ British Antarctic Survey, Natural Environment Research Council, Madingley Road, Cambridge CB3 OET, England
}

\begin{abstract}
A perturbation method is used to analyse the stability of a thin till layer overlain by a deep ice layer. Ice is modelled as a linearly viscous fluid, while the till viscosity has power-law dependence on stress and effective pressure. A linearized set of equations yields descriptions of the coupling of the ice flow with the sediment flow and reveals parameter ranges where the till-perturbation amplitude can grow. This sheet-flow instability is an essential part of any theory of drumlin formation and shows that viscous models of till have the ability to explain typical deforming-bed features. This is of great significance for large-scale ice-sheet modelling.
\end{abstract}

\section{NOTATION}

\begin{tabular}{|c|c|}
\hline Variable & Meaning \\
\hline$a, b$ & Till-flow model indices \\
\hline$k$ & Wave number \\
\hline$p$ & Ice pressure \\
\hline$p_{\mathrm{e}}$ & Effective pressure \\
\hline$\hat{p}_{\mathrm{c}}$ & $p_{\mathrm{e} 0}-\alpha D_{0}$ \\
\hline$q$ & Till flux \\
\hline$t$ & Time \\
\hline$(u, w)$ & Velocity \\
\hline$\hat{u}_{1}(x, z)$ & $u_{1}(x, z)-D_{1} \partial_{z} U$ \\
\hline$(x, z)$ & Position \\
\hline$A_{\mathrm{d}}$ & Rate factor for till \\
\hline$C^{\alpha}, C^{\beta}$ & Fourier coefficients \\
\hline$D$ & Till thickness \\
\hline$D^{\alpha}, D^{\beta}$ & Fourier coefficients for $D$ \\
\hline$D^{c}$ & $\left(\left(D^{\alpha}\right)^{2}+\left(D^{\beta}\right)^{2}\right)^{\frac{1}{2}}$ \\
\hline$F_{\mathrm{u}}$ & Velocity function for till deformation \\
\hline$F_{\mathrm{q}}$ & Flux function for till deformation \\
\hline$M$ & Migration velocity \\
\hline$P^{\alpha}, P^{\beta}$ & Fourier coefficients for $p$ \\
\hline$Q_{\mathrm{t}}, Q_{\mathrm{n}}, Q_{\mathrm{D}}$ & Derivatives of $F_{\mathrm{q}}$ \\
\hline$R_{\mathrm{t}}, R_{\mathrm{n}}, R_{\mathrm{D}}$ & Derivatives of $F_{\mathrm{u}}$ \\
\hline$T^{\mathrm{t}}, T^{\mathrm{n}}$ & $\begin{array}{l}\text { Tangential, normal traction at sediment } \\
\text { surface }\end{array}$ \\
\hline$U$ & Reference (simple shear) velocity field \\
\hline $\begin{array}{l}\alpha=\left(\rho_{\mathrm{w}}-\rho_{\mathrm{i}}\right) g \\
\beta=(1-\phi)\end{array}$ & Interfacial $p_{\mathrm{e}}$ static gradient \\
\hline$\left(\rho_{\mathrm{s}}-\rho_{\mathrm{w}}\right) g$ & Internal $p_{\mathrm{e}}$ static gradient \\
\hline$\gamma$ & $\alpha+\beta$ \\
\hline$\varepsilon$ & Expansion parameter \\
\hline$\eta$ & Ice viscosity \\
\hline$\phi$ & Sediment porosity \\
\hline$\omega$ & Phase \\
\hline
\end{tabular}

\section{INTRODUCTION}

Drumlins are mounds of sediments produced by the action of ice sheets and glaciers. They range in size from a few metres to tens of metres high and range in length from tens of metres to kilometres. Typically, drumlins have blunt upstream and occasionally downstream faces. The problem of explaining drumlin formation, which has troubled glacial geologists for over a century, was re-invigorated in the 1980 s when it was realized that sediment deformation could explain many of the structures found within drumlins (Boulton, 1987) and it was hypothesized that, if subglacial sediment were essentially deforming as a viscous fluid, then drumlin formation might be a problem explicable using fluid-dynamical principles (Boulton and Hindmarsh, 1987). This has important consequences, because the same physics which explain drumlin formation can also explain icestream lubrication by deforming sediment, as observed beneath Ice Stream B (Blankenship and others, 1986). The ability to explain drumlin formation can thus be seen as a key test for any theory of subglacial sediment transport and, if subglacial sediment deformation is an important sediment transport route, the study of drumlins provides information about the large-scale flow of sediment, information which cannot be measured in the laboratory for practical reasons (Hindmarsh, 1997).

Recently, the blunt faces of drumlins have been explained as shocks (Hindmarsh, 1996, in press a) and a nonlinear kinematical theory of drumlin formation has been developed. However, while a certain proportion of drumlins are obviously moulded from pre-existent relief, there is a sufficient proportion of drumlins composed of the same material as surrounding till (subglacial sediment) fields to suggest that drumlin formation may be an instability in a viscous flow of a till sheet. This idea has long been debated amongst glacial geologists. In this paper, we examine the stability of a till sheet at wavelengths longer than the depth of the deforming till but shorter than the ice-sheet thickness; this is a typical drumlin wavelength.

In general, the flow of two shearing layers of different viscosities but the same density is stable. In this case, the viscosity of the till layers is affected by the normal traction applied by the ice. Moreover, till discharge does not increase 
monotonically with till thickness (for a given stress) but decreases, owing to increased effective pressures (Hindmarsh, in press a). On its own, this does not lead to unstable thickening of the till layer but the interaction of this property with the ice flow might. This is investigated in the present paper. It is found that under certain conditions, but not all conditions, that unstable thickening does occur; sheet flow is not stable. A feature which emerges is that, in general, till bed forms migrate. This is not something which has been extensively discussed in the literature and indeed it is hard to see how it might be inferred from formerly glaciated areas. Rock-cored drumlins might at first sight appear to militate against this idea but, since so many drumlins are not rock-cored, one has to regard rock-cored drumlins as atypical in genesis and therefore in dynamics. It is hard to imagine a viscous theory which does not in general predict migration.

The ice-flow model is a variant of the perturbation approach developed by Nye $(1969,1970)$ and Kamb (1970), called here the NK solution, and, since the ice is in a state of simple shear at large distances from the bed, also bears some relation to the analysis by Morland (1976a, b); it is not, however, a gravity-driven flow. Rather than slipping over a perfectly smooth, fixed bed, it is fixed to a thin deforming fluid, the till, whose stress fields can be computed using lubrication-theory approximations (known in glaciology as the thin-till approximation (Alley, 1989)). The evolution of the till profile can then be computed and it is found that in certain parameter ranges the surface relief grows, although of course the till volume remains constant. This is the principal result of the paper; ice flowing over a thin till sheet can promote unstable amplification of relief. This is a necessary component of a theory of drumlinization if it is believed that not all drumlins are created by moulding of pre-existent relief.

This analysis is based upon the viscous flow laws for till where strain rate is proportional to the shear stress to some low power and inversely proportional to the effective pressure to some low power (Boulton and Hindmarsh, 1987). The relevant paper was written in the context of six decades of mechanical research which showed that on the small scale sediment was plastic. At Breiðamerkurjökull, the ice overriding deforming sediment does not build up and then slip; en masse, it does not behave plastically. A viscous law, while undoubtedly failing to represent failure, represents the simplest model compatible with large-scale observations. In the same way as observations of dislocations in ice (an event akin to plastic failure) are never held to militate against the validity of viscous descriptions of ice, there appears to me to be no contradiction between laboratory experiments which demonstrate plastic behaviour and the posing of a viscous law which applies on a larger scale (Bahr and Rundle, 1996; Hindmarsh, 1997). Recent studies, for example, by Kamb (1991) and Iverson and others (1995), which question the existence of local viscous behaviour, do not explicitly address the question of what an appropriate large-scale law might be. On the other hand, to be of any use, a large-scale viscous law must be shown to be able to reproduce observed behaviour. This paper shows that the viscous flow of till, when coupled with ice flow, can create an instability which appears to be necessary in the formation of drumlins (Hindmarsh, in press a). Actually, it also shows that power-law indices as high as 10 (which some might call plastic behaviour) can create drumlins.

\section{STOKES EQUATIONS AND BOUNDARY CONDITIONS FOR SHEAR OVER A MOBILE BED}

The solution domain is an ice-sheet half-space overlying a deforming till layer of finite thickness. The ice and the till are supposed to be fully coupled, so that velocities are continuous across the interface. A shear stress is applied to the ice at a large distance from the bed, which sets up a simple shear in the ice and causes the till to deform. In the base case, the till surface is flat, meaning that a simple shear exists in the ice and that the till deforms with uniform velocity at its upper surface. Small periodic variations in the till surface profile are then introduced, which cause the simple shear in the ice flow to be modified, according to a variant of the NK perturbation of the Stokes equations. Flow in the till is still computed according to lubrication theory principles, implying that the wavelength of the disturbances be greater than the deforming layer thickness. The perturbation to the ice flow implies perturbations to the tangential and normal tractions applied to the till, causing the till flow to be perturbed, and it is the distribution of these which determines whether the till relief grows or decays.

Consider the ice to be occupying a half-space periodic in the horizontal direction in the domain $x=[0,2 \pi / k]$ and $z \geq 0$, where $k$ is a wave number. We can generate a zerothorder solution by assuming that the bed is flat and that the till is thus of uniform thickness $D=D_{0}$, with the top of the till at $z=0$. Let us suppose that a traction $T_{0}^{\mathrm{t}}$ is applied to a horizontal surface on the ice at a distance very far from the bed. This sets up a simple shear in the ice and in the till and also creates a horizontal velocity in the ice at the bed $z=0$. We find that the zeroth-order velocity is

$$
u_{0}=U_{\mathrm{b}}+z \partial_{\mathrm{z}} U, \partial_{\mathrm{z}} U=T_{0}^{\mathrm{t}} / \eta, U_{\mathrm{b}}=F_{\mathrm{u}}\left(T_{0}^{\mathrm{t}}, p_{\mathrm{e} 0}, D_{0}\right)
$$

where $p_{\mathrm{e} 0}$ is the interfacial effective pressure and $F_{\mathrm{u}}$ is a function to be discussed later. This shear implies a flux of till given by

$$
q=F_{q}\left(T_{0}^{\mathrm{t}}, p_{\mathrm{e} 0}, D_{0}\right)
$$

In this analysis, the effective pressure is a free parameter, which in reality is determined by the subglacial hydraulic system. In this study, horizontal scales are assumed to be sufficiently small that pressure gradients are hydrostatic; there are no horizontal water-pressure gradients. The physics of this has been discussed by Hindmarsh (1996, in press).

Following Nye (1969, 1970) and Kamb (1970), we perturb the zeroth-order solution of ice flow by introducing some undulations into the bed. There is a difference here compared with the NK solution, because we have a base solution with simple shear (and thus no possible problems with the Stokes paradox (Fowler, 1981)), the situation is in some ways analogous to the gravity-driven flows computed by Morland (1976a, b). Moreover, we follow Nye (1970) by working in a perturbed coordinate system where $z$ is measured from above the bed. This transform is covered in more detail in the Appendix. 
The perturbation expansion of field values in the ice in this transformed coordinate system is

$u=U_{\mathrm{b}}+\partial_{z} U\left(z+\varepsilon D_{1}\right)+\varepsilon u_{1}(x, z)+O\left(\varepsilon^{2}\right)$,

$w=\varepsilon w_{1}(x, z)+O\left(\varepsilon^{2}\right), \quad p=\varepsilon p_{1}(x, z)+O\left(\varepsilon^{2}\right)$,

$D=D_{0}+\varepsilon D_{1}(x)+O\left(\varepsilon^{2}\right)$

with basal boundary perturbation expressions

$$
T^{\mathrm{n}}=T_{0}^{\mathrm{n}}+\varepsilon T_{1}^{\mathrm{n}}, \quad T^{\mathrm{t}}=T_{0}^{\mathrm{t}}+\varepsilon T_{1}^{\mathrm{t}}
$$

and it is shown in the Appendix that we obtain the following first-order field equations

$$
\begin{aligned}
\eta \nabla^{2} u_{1} & =\partial_{x} p_{1}+\partial_{x}^{2} D_{1} \partial_{z} U, \\
\eta \nabla^{2} w_{1} & =\partial_{z} p_{1}, \\
\partial_{x} u_{1}+\partial_{z} w_{1} & =\partial_{z} U \partial_{x} D_{1}
\end{aligned}
$$

with boundary relations

$$
T_{1}^{\mathrm{t}}=\eta\left(\frac{\partial u_{1}}{\partial z}+\frac{\partial w_{1}}{\partial x}\right), \quad T_{1}^{\mathrm{n}}=2 \eta\left(\frac{\partial u_{1}}{\partial x}-\partial_{z} U \partial_{x} D_{1}\right)+p_{1}
$$

where the normal traction convention is compressive-positive. If we define a new function

$$
\hat{u}_{1}(x, z)=u_{1}(x, z)-D_{1} \partial_{z} U,
$$

we can retrieve the more familiar set of NK field equations

$$
\begin{aligned}
\eta \nabla^{2} \hat{u}_{1} & =\partial_{x} p_{1}, \\
\eta \nabla^{2} w_{1} & =\partial_{z} p_{1}, \\
\partial_{x} \hat{u}_{1}+\partial_{z} w_{1} & =0
\end{aligned}
$$

but now with boundary relations

$$
\begin{aligned}
& T_{1}^{\mathrm{t}}=\eta\left(\frac{\partial \hat{u}_{1}}{\partial z}+\frac{\partial w_{1}}{\partial x}\right), \\
& T_{1}^{\mathrm{n}}=-\sigma_{z z_{1}}=\tau_{x x_{1}}+p_{1}=2 \eta\left(\frac{\partial \hat{u}_{1}}{\partial x}\right)+p_{1} .
\end{aligned}
$$

The first-order basal velocity is computed using a linearization of the basal velocity relationship (1), so we can write $u_{1}(x, z=0)=R_{\mathrm{t}} T_{1}^{\mathrm{t}}+R_{\mathrm{n}} T_{1}^{\mathrm{n}}+R_{\mathrm{D}} D_{1}$,

$$
R_{\mathrm{t}}=\partial F_{\mathrm{u}} / \partial \tau, \quad R_{\mathrm{n}}=\partial F_{\mathrm{u}} / \partial p_{\mathrm{e}}, \quad R_{\mathrm{D}}=\partial F_{\mathrm{u}} / \partial D
$$

where the derivatives are evaluated around the zeroth-order state. We also need to satisfy the first-order kinematical condition

$$
\partial_{\mathrm{t}} D_{1}+U_{\mathrm{b}} \partial_{x} D_{1}=w_{1}
$$

where $\partial_{t} D_{1}$ (by construction zero in the NK formulation) is given by the linearized till-conservation equation

$$
\begin{aligned}
\partial_{\mathrm{t}} D_{1} & =-Q_{\mathrm{t}} \partial_{x} T_{1}^{\mathrm{t}}-Q_{\mathrm{n}} \partial_{x} T_{1}^{\mathrm{n}}-Q_{\mathrm{D}} \partial_{x} D_{1}, \\
Q_{\mathrm{t}} & =\partial F_{\mathrm{q}} / \partial \tau, \quad Q_{\mathrm{n}}=\partial F_{\mathrm{q}} / \partial p_{\mathrm{e}}, \quad Q_{\mathrm{D}}=\partial F_{\mathrm{q}} / \partial D .
\end{aligned}
$$

Some readers may feel these perturbations look strange as they appear to lack the expected products of zeroth- and first-order terms. In fact, all the zeroth-order terms are in the coefficients $R_{\mathrm{t}, \mathrm{n}, \mathrm{D}}, Q_{\mathrm{t}, \mathrm{n}, \mathrm{D}}$. A quick way of verifying the validity of the method is to remember that a linearization is simply a Taylor expansion; the Taylor coefficients here are simply $R_{\mathrm{t}, \mathrm{n}, \mathrm{D}}, Q_{\mathrm{t}, \mathrm{n}, \mathrm{D}}$.

The velocities and fluxes are computed following Alley (1989) and Hindmarsh (in press a). Till flux can arise either from internal deformation within the till or from till sliding over the base. When considering internal deformation, the strain rate in the till is given by a double power-law rheology (Boulton and Hindmarsh, 1987)

$$
\frac{\partial u}{\partial z}=\frac{A_{\mathrm{d}} \tau^{a}}{p_{\mathrm{e}}(z)^{b}}=\frac{A_{\mathrm{d}} \tau^{a}}{\left(p_{\mathrm{e} 0}+\alpha\left(D-D_{0}\right)+\beta\left(D-D_{0}-z\right)\right)^{b}}
$$

where $A_{\mathrm{d}}$ is a rate factor and $a$ and $b$ are parameters. Then, defining

$$
\hat{p}_{c}=p_{\mathrm{e} 0}-\alpha D_{0}
$$

whence after integrating with respect to $z$ over the interval $-D_{0} \leq z \leq D-D_{0}$ we find

$$
u=F_{\mathrm{u}}=\frac{A_{\mathrm{d}} \tau^{a}}{\beta(b-1)}\left(\left(\hat{p}_{\mathrm{c}}+\alpha D\right)^{1-b}-\left(\hat{p}_{\mathrm{c}}+\gamma D\right)^{1-b}\right),
$$

and the flux is given by

$$
\begin{aligned}
q=F_{\mathrm{q}}= & \frac{A_{\mathrm{d}} \tau^{a}}{(b-1)(b-2) \beta^{2}} \times \\
& \left(\left(\hat{p}_{\mathrm{c}}+\alpha D\right)^{2-b}-\left(\hat{p}_{\mathrm{c}}+\gamma D\right)^{1-b}\left(\hat{p}_{\mathrm{c}}+\Omega D\right)\right)
\end{aligned}
$$

where

$$
\Omega=\alpha+\beta(b-1)=\gamma+\beta(b-2) .
$$

In these formulae

$$
\alpha=\left(\rho_{\mathrm{w}}-\rho_{\mathrm{i}}\right) g, \beta=(1-\phi)\left(\rho_{\mathrm{s}}-\rho_{\mathrm{w}}\right) g, \gamma=\alpha+\beta
$$

where $g$ is the acceleration due to gravity, $\rho_{\mathrm{w}}, \rho_{\mathrm{i}}$ and $\rho_{\mathrm{s}}$ are the densities of ice, water and sediment grains, and $\phi$ is the porosity of the sediment. We use the values $9.81 \mathrm{~m} \mathrm{~s}^{-2}$, $1.0 \mathrm{Mg} \mathrm{m}^{-3}, 0.917 \mathrm{Mg} \mathrm{m}^{-3}, 2.7 \mathrm{Mg} \mathrm{m}^{-3}$ and 0.2 , respectively, in this paper. These formulae are derived in exactly the same way as the equivalent relationships in Alley (1989) but account for statically induced vertical interfacial effective pressure gradients arising from the density difference between water and ice (Hindmarsh, 1996). Under static assumptions, an increase in elevation causes an increase in effective pressure because water pressure decreases more rapidly than does ice pressure as one ascends. Alley's formulae may be retrieved by setting $\alpha=0$.

We now turn our attention to till-bed sliding. If we use the viscous-type sliding law proposed by Hindmarsh (1996), we find that

$$
u(D)=\frac{A_{\mathrm{s}} \tau^{\mathrm{c}}}{\left(\hat{p}_{\mathrm{c}}+\gamma D\right)^{\mathrm{d}}}, q=\frac{A_{\mathrm{s}} \tau^{\mathrm{c}} D}{\left(\hat{p}_{\mathrm{c}}+\gamma D\right)^{\mathrm{d}}} .
$$

Sliding and deformation do not occur together in this study. We note that the more complicated derivatives are given by

$$
\begin{aligned}
R_{\mathrm{n}}= & \frac{\partial u}{\partial p_{\mathrm{e}}}=A_{\mathrm{d}} \frac{\tau^{a}}{\beta}\left(\left(\hat{p}_{\mathrm{c}}+\gamma D\right)^{-b}-\left(\hat{p}_{\mathrm{c}}+\alpha D\right)^{-b}\right), \quad(19 \mathrm{a}) \\
R_{\mathrm{D}}= & \frac{\partial u}{\partial D}=A_{\mathrm{d}} \frac{\tau^{a}}{\beta}\left(\gamma\left(\hat{p}_{\mathrm{c}}+\gamma D\right)^{-b}-\alpha\left(\hat{p}_{\mathrm{c}}+\alpha D\right)^{-b}\right),(19 \mathrm{~b}) \\
Q_{\mathrm{n}}= & \frac{\partial q}{\partial p_{\mathrm{e}}}=\frac{A_{\mathrm{d}} \tau^{a}}{\beta^{2}(b-1)(b-2)} \times \\
& \left(\frac{(b-1)\left(\hat{p}_{\mathrm{c}}+\Omega D\right)-\left(\hat{p}_{c}+\gamma D\right)}{\left(\hat{p}_{\mathrm{c}}+\gamma D\right)^{b}}-\frac{(b-2)}{\left.\left(\hat{p}_{\mathrm{c}}+\alpha D\right)^{b-1}\right)}\right) \\
Q_{\mathrm{d}}= & \frac{\partial q}{\partial D}=\frac{A_{\mathrm{d}} \tau^{a}}{\beta^{2}(b-1)}\left(\frac{\alpha \hat{p}_{\mathrm{c}}+\gamma \Omega D}{\left(\hat{p}_{\mathrm{c}}+\gamma D\right)^{b}}-\frac{\alpha}{\left(\hat{p}_{\mathrm{c}}+\alpha D\right)^{b-1}}\right) .
\end{aligned}
$$

A significant point is that we have assumed that the shear stress is constant within the till. At wavelengths comparable with the deforming till thickness, this approxima- 
tion breaks down, and gradients in the till thickness and the applied normal stress cause additional flow effects. Our analysis, therefore, is only valid for wavelengths between the thickness of the till layer and the thickness of the ice layer. This is the relevant length scale as most drumlins are of this size.

\section{SOLUTION}

Let us suppose that the bed profile is given by

$$
D_{1}(t)=D_{1}^{\alpha}(t) \sin k x+D_{1}^{\beta}(t) \cos k x
$$

where $D_{1}^{\alpha}(t), D_{1}^{\beta}(t)$ are scalar functions of $t$. The solution to the field Equations (6) is

$$
\begin{aligned}
& w_{1}=\exp (-k z)(\left\{C^{\alpha}+\frac{P^{\alpha}}{2 \eta}\left(\frac{1}{k}+z\right)\right\} \cos k x . \\
&+\left.\left\{C^{\beta}+\frac{P^{\beta}}{2 \eta}\left(\frac{1}{k}+z\right)\right\} \sin k x\right), \\
& \hat{u}_{1}=\exp (-k z)\left(\left\{C^{\alpha}+\frac{P^{\alpha}}{2 \eta} z\right\} \sin k x\right.\left.\left\{C^{\beta}+\frac{P^{\beta}}{2 \eta} z\right\} \cos k x\right), \\
& p_{1}=\exp (-k z)\left(P^{\alpha} \cos k x+P^{\beta} \sin k x\right)
\end{aligned}
$$

where the coefficients $C^{\alpha}, P^{\alpha}, C^{\beta}, P^{\beta}$ are determined by the basal boundary conditions. At the base, $z=0$, we compute the tangential traction and its gradient as follows

$$
T_{1}^{t}=\eta\left(\partial_{z} \hat{u}_{1}+\partial_{x} w_{1}\right)=2 \eta k\left(C^{\beta} \cos k x-C^{\alpha} \sin k x\right),
$$

$$
\partial_{x} T_{1}^{t}=-2 \eta k^{2}\left(C^{\beta} \sin k x+C^{\alpha} \cos k x\right)
$$

and the normal traction and its gradient as

$$
\begin{aligned}
T_{1}^{n} & =p_{1}+2 \eta \partial_{x} \hat{u}_{1} \\
& =\left(2 \eta k C^{\alpha}+P^{\alpha}\right) \cos k x+\left(2 \eta k C^{\beta}+P^{\beta}\right) \sin k x, \\
\partial_{x} T_{1}^{n} & =-k\left(\left(2 \eta k C^{\alpha}+P^{\alpha}\right) \sin k x-\left(2 \eta k C^{\beta}+P^{\beta}\right) \cos k x\right)
\end{aligned}
$$

while the interfacial velocities are given by

$w_{1}=\left(C^{\alpha}+\frac{P^{\alpha}}{2 k \eta}\right) \cos k x+\left(C^{\beta}+\frac{P^{\beta}}{2 k \eta}\right) \sin k x$,

$\hat{u}_{1}=C^{\alpha} \sin k x-C^{\beta} \cos k x$.

Substitution of Equations (20), (22a), (22b), (23a), 23b), (24a) and (24b) into Equation (8) and also into Equation (10), where we have eliminated $\partial_{t} D_{1}$ by using Equation (11) and subsequent multiplication of each of these equations by $\sin k x$ and separately by $\cos k x$ followed by integration over the horizontal domain yields the following system of four equations for $C^{\alpha}, C^{\beta}, P^{\alpha}, P^{\beta}$ :

$$
\begin{gathered}
{\left[\begin{array}{cccc}
1+2 k \eta R_{\mathrm{t}} & -2 k \eta R_{\mathrm{n}} & 0 & -R_{\mathrm{n}} \\
2 k \eta R_{\mathrm{n}} & 1+2 k \eta R_{\mathrm{t}} & R_{\mathrm{n}} & 0 \\
1-2 k^{2} \eta Q_{\mathrm{t}} & 2 k^{2} \eta Q_{\mathrm{n}} & 1 / 2 k \eta & k Q_{\mathrm{n}} \\
-2 k^{2} \eta Q_{\mathrm{n}} & 1-2 k^{2} \eta Q_{\mathrm{t}} & -k Q_{\mathrm{n}} & 1 / 2 k \eta
\end{array}\right]\left[\begin{array}{c}
C^{\alpha} \\
C^{\beta} \\
P^{\alpha} \\
P^{\beta}
\end{array}\right]} \\
=\left[\begin{array}{c}
R_{\mathrm{d}} D_{1}^{\alpha} \\
-R_{\mathrm{d}} D_{1}^{\beta} \\
k\left(U_{\mathrm{b}}-Q_{\mathrm{d}}\right) D_{1}^{\alpha} \\
-k\left(U_{\mathrm{b}}-Q_{\mathrm{d}}\right) D_{1}^{\beta}
\end{array}\right] .
\end{gathered}
$$

Solutions are discussed below.

Superposability is easy to demonstrate by expanding ar- bitrary (differentiable) bed shapes in a Fourier series. The above equation holds for each bed frequency, and solutions for the stress and velocity fields may be obtained for each wave number which depend only on the bed geometry at that wave number. This also applies to the linearized till thickness evolution equation discussed below, meaning that evolution equations may be obtained separately for each mode.

The linearized till conservation equation is

$$
\partial_{t} D_{1}=-Q_{\mathrm{t}} \partial_{x} T_{1}^{\mathrm{t}}-Q_{\mathrm{n}} \partial_{x} T_{1}^{\mathrm{n}}-Q_{\mathrm{d}} \partial_{x} D_{1} .
$$

Using relations (22b) and (23b), we can show that the phase and anti-phase mode-evolution equations are

$$
\begin{aligned}
& \dot{D}_{1}^{\mathrm{e}}=2 k^{2} \eta Q_{\mathrm{t}} C^{\beta}+k Q_{\mathrm{n}}\left(2 \eta k C^{\alpha}+P^{\alpha}\right)+Q_{\mathrm{d}} k D_{1}^{\beta}, \\
& \dot{D}_{1}^{\mathrm{o}}=2 k^{2} \eta Q_{\mathrm{t}} C^{\alpha}-k Q_{\mathrm{n}}\left(2 \eta k C^{\beta}+P^{\beta}\right)-Q_{\mathrm{d}} k D_{1}^{\alpha} .
\end{aligned}
$$

This solution represents a sine wave growing or shrinking in amplitude and translating. The rate of growth of the bed perturbation $\left|D_{1}^{\mathrm{c}}\right|$ is

$$
\left|D_{1}^{\mathrm{c}}\right|\left|\dot{D}_{1}^{\mathrm{c}}\right|=\left(2 k \eta Q_{t} C^{\alpha}+k\left(2 k \eta C^{\beta}+P^{\alpha}\right) Q_{n}\right) D_{1}^{\alpha},
$$

and we can use the idea of the growth-rate constant

$$
\frac{\left|\dot{D}_{1}^{\mathrm{c}}\right|}{\left|D_{1}^{\mathrm{c}}\right|}=\frac{\left(2 k \eta Q_{\mathrm{t}} C^{\alpha}+k\left(2 k \eta C^{\beta}+P^{\beta}\right) Q_{\mathrm{n}}\right) D_{1}^{\alpha}}{\left|D_{1}^{\mathrm{c}}\right|}
$$

This analysis shows that an incipient drumlin will move. The phase $\omega$ is given by

$$
\omega=\arctan \left(\frac{D_{1}^{\beta}}{D_{1}^{\alpha}}\right)
$$

and the migration velocity $M$ of a sine wave with wave number $k$ is given by $-\dot{\omega} / k$. It can readily be shown that

$$
\dot{\omega}=\frac{1}{\left(D_{1}^{c}\right)^{2}}\left(D_{1}^{\alpha} \dot{D}_{1}^{\beta}-D_{1}^{\beta} \dot{D}_{1}^{\alpha}\right)
$$

and in this case the velocity $M$ of a sinusoidal till wave with wave number $k$ is given by

$$
\begin{aligned}
M= & \frac{Q_{\mathrm{d}}-2 k Q_{t} \eta\left(C^{\alpha} D_{1}^{\alpha}-C^{\beta} D_{1}^{\beta}\right)}{\left(D_{1}^{\mathrm{c}}\right)^{2}} \\
& +Q_{n} \frac{\left(\left(2 \eta k C^{\beta}+P^{\beta}\right) D_{1}^{\alpha}+\left(2 k \eta C^{\alpha}+P^{\alpha}\right) D_{1}^{\beta}\right)}{\left(D_{1}^{\mathrm{c}}\right)^{2}} .
\end{aligned}
$$

We call this the migration velocity. Where there is no ice-till coupling, the migration velocity is equal to the kinematic wave velocity $Q_{\mathrm{d}}$. It can be shown that $M=$ $Q_{\mathrm{d}}+(\mathrm{d} q / \mathrm{d} f)\left(\partial_{x}^{2} f / \partial_{x}^{2} D\right)$, where $f$ is a field variable other than $D$, such as $T_{\mathrm{t}}$. This result generalizes to an arbitrary number of field variables. Thus, we do not expect the migration velocity to be equal to the kinematic wave velocity when $Q_{\mathrm{t}}$ or $Q_{\mathrm{n}}$ are non-zero - where there are gradients in the field variables, the elevation of a kinematic wave is not constant. Owing to the relationship between the migration velocity and the kinematic wave velocity, it is hard to conceive a viscous theory which does not have migrating drumlins.

\section{EXAMPLES AND APPLICATIONS}

Without loss of generality, since we are considering cases of individual Fourier modes, we set $D^{\beta}=0$, which considerably simplifies the solutions given below. It is straightfor- 
ward to show that the force exerted by tangential traction must sum to zero at this order, and the additional drag, which is a second-order quantity (Fowler, 1981), is obtained from the normal traction

$$
\tau_{\mathrm{b}}=\frac{k}{2}\left(2 \eta k\left(C^{\alpha} D_{1}^{\alpha}-C^{\beta} D_{1}^{\beta}\right)+P^{\alpha} D_{1}^{\alpha}-P^{\beta} D_{1}^{\beta}\right) .
$$

We find that the solutions to the matrix Equation (25) for all the $Q$ zero, implying a bed of fixed geometry, are

$$
\left[\begin{array}{c}
C^{\alpha} \\
C^{\beta} \\
P^{\alpha} \\
P^{\beta}
\end{array}\right]=\frac{D_{1}^{\alpha}}{1+2 k \eta R_{\mathrm{t}}}\left[\begin{array}{c}
c R_{\mathrm{d}} \\
-2 R_{\mathrm{n}} k^{2} \eta U_{\mathrm{b}} \\
4 \eta^{2} k^{3} U_{\mathrm{b}} R_{\mathrm{t}}+2 k \eta\left(k U_{\mathrm{b}}-R_{\mathrm{d}}\right) \\
4 \eta^{2} k^{3} U_{\mathrm{b}} R_{\mathrm{n}}
\end{array}\right] .
$$

If all the $R$ are zero (no slip) then $C^{\alpha}, C^{\beta}$ are zero and we find that $P^{\alpha}, P^{\beta}=0$ since $U_{\mathrm{b}}$ is zero for a fixed bed. Nothing happens at this order of the approximation. If the basal conditions are very slippery ( $R_{\mathrm{t}}$ very large) but the other $R$ remain zero then we retrieve the $\mathrm{NK}$ solution $C^{\alpha}=0$, $P^{\alpha}=2 \eta k^{2} U_{\mathrm{b}} D_{1}^{\alpha}$. It is not obvious that we should get this but nonetheless we do.

Note that non-zero anti-phase components $C^{\beta}, P^{\beta}$ only occur when there is a dependence of the basal velocity on the effective pressure. Moreover, when the bed is not mobile, the basic Equation set (25) and expressions (24a) and (24b) ensure that there is no anti-phase structure in the normal velocity at the interface nor in the normal traction.

The full set of equations, with non-zero $Q_{\mathrm{t}}, Q_{\mathrm{n}}, Q_{\mathrm{D}}$ do possess solutions in rationals of polynomials involving no radicals, which are nonetheless too complicated to peruse. Numerical solutions involving direct inversion of Equation (25) are readily obtainable. Our main question concerns the possible existence of unstable Fourier modes which cause bed relief to grow. We now show that they must be associated with the existence of anti-phase structure. In consequence, since anti-phase structure is excited by the dependence of the deforming-bed viscosity on the effective pressure and through this, on the applied normal traction, we see that it is the dependence of deforming sediment rheology on effective pressure which causes unstable growth of relief.

First, we note that the rate of change following the wave is given by $\mathrm{d} D /\left.\mathrm{d} t\right|_{\mathrm{w}}=\partial_{\mathrm{t}} D+M \partial_{x} D$. Since, at the maximum, $\partial_{x} D=0$, the rate of change of the maximum point following the maximum is simply $\partial_{\mathrm{t}} D$. Consider now the bed-evolution equation

$$
\partial_{\mathrm{t}} D_{1}=-Q_{\mathrm{t}} \partial_{x} T_{1}^{\mathrm{t}}-Q_{\mathrm{n}} \partial_{x} T_{1}^{\mathrm{n}}-Q_{\mathrm{d}} \partial_{x} D_{1} .
$$

The elevation maximum occurs at $x=\pi / 2 k$. At this point, relationship (22b) shows that (in the absence of anti-phase structure) that the first term on the righthand size is zero, as is the third term. In general, a zero $R_{\mathrm{n}}$ implies a zero $Q_{\mathrm{n}}$, but even if that were not the case, a more detailed examination shows that for $Q_{\mathrm{n}}<0$, the usual case, the sediment relief will decline. The feature which causes the sediment relief to evolve is a non-zero $R_{\mathrm{n}}$.

There are a large number of factors which influence the evolution of the bed profile; the zeroth-order stresses $\tau_{0}$ and $p_{\mathrm{e} 0}$, the sediment deformation and sliding rate factors, and the various indices. Since it is evident that there is a direct dependence of the perturbation on the basal velocity $U_{\mathrm{b}}$, instead of varying the rate factors as a parameter, we vary the velocity $U_{\mathrm{b}}$ as a parameter and choose the rate factor to give the required velocity.

Approximately 240000 calculations considering internal deformation have been carried out, comprising the direct product of the following seven-dimensional parameter space: $D_{0} \in[0.1,100] \mathrm{m}, U_{\mathrm{b}} \in[1,1000] \mathrm{ma}^{-1}$ the wavelength $2 \pi / k \in[10,1000], p_{\mathrm{e} 0} \in\left[2 \times 10^{3}, 10^{5}\right] \mathrm{Pa}, \tau_{0} \in[2 \times$ $\left.10^{3}, 10^{5}\right] \mathrm{Pa},(a, c) \in[1,10]$ and $(b, d) \in[1.02,10]$. Such a large exploration of parameter space would not have been possible using numerical solutions of the full equations. The main aim has been to identify the regions of parameter space which encourage amplification of relief. Figure 1 shows the proportion of positive growth rates for each parameter value. All cases for a particular parameter value are considered; for example, if the effective pressure is 0.002 , into this bin goes every case where this is true.

Thus, the proportion of positive growth rates increases

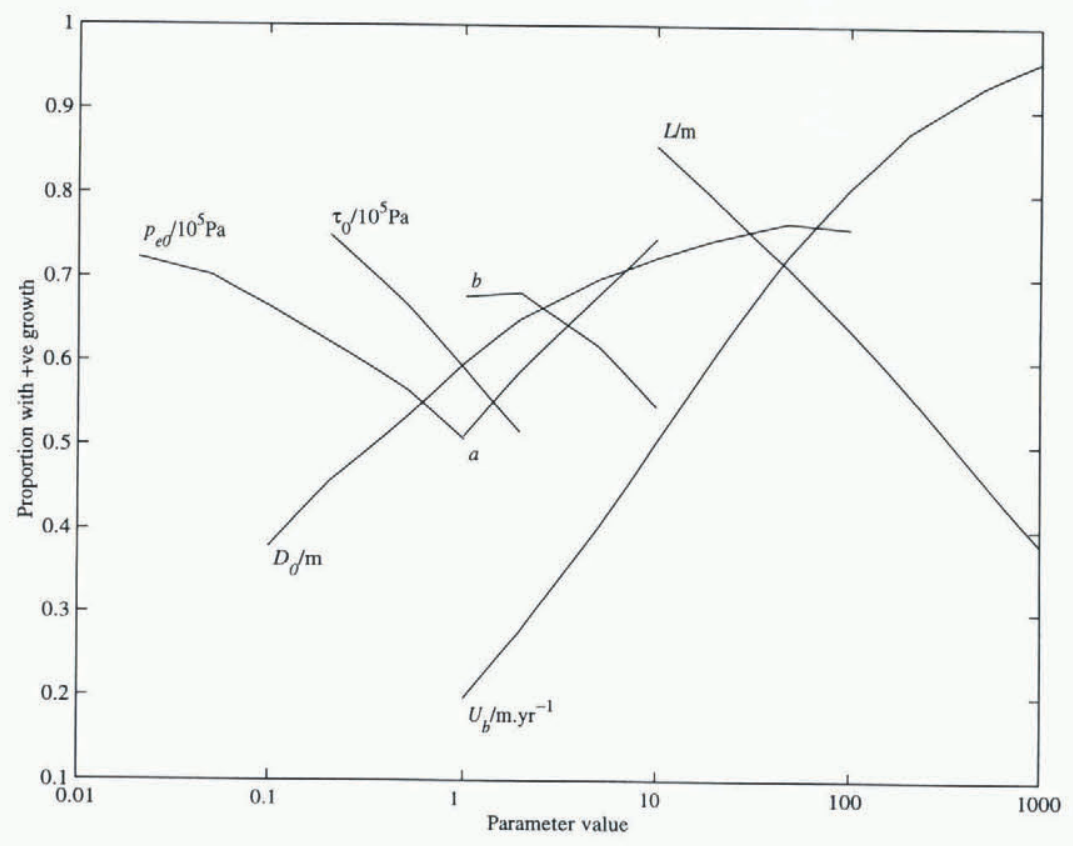

Fig. 1. Proportion of cases where the growth rate is positive, plotted using each parameter as the independent variable. Note that a starts just where $p_{\mathrm{e}}$ finishes. Parameter units are indicated on the main plot. 
with basal velocity, declines with perturbation wavelength, and shows a non-monotone relationship with till thickness, reaching a maximum at till thicknesses around $50 \mathrm{~m}$. This need not be true for any particular parameter value. The proportion decreases with effective pressure and shear stress, increases with shear-stress index and has a nonmonotone relationship on the effective pressure index.

Individual cases are not so clear cut. Figure 2a shows how growth rate depends on the till thickness and the basal velocity for the indicated values. For low values of till thickness and basal velocity, growth rates are negative, and there is a monotone increasing dependence on basal velocity but a non-monotone dependence on till thickness, but with growth rates remaining positive. For a rather more non-linear flow law, Figure 2b shows a simpler dependence on till thickness and a different pattern of negative growth rates. Figure $2 \mathrm{c}$ has basal velocity and wavelength as independent variables. High wavelength means negative growth rates for low velocities. Figure 2d shows that high effective pressures can suppress drumlin formation for small till thicknesses and that there is a non-monotone dependence of growth rate upon effective pressure.

In general, these cases suggest that high velocity and greater till thickness encourage drumlin formation. The result that is encouraged at small wavelengths is dependent on the assumption that the thin till approximation holds good. Drumlins presently found in very thin till sequences may therefore be erosional remnants, if cases exist where longitudinal stress gradients within the till suppress drumlin formation. One curious feature is that positive growthrate constants are very much larger in magnitude than are negative ones and are high enough to suggest that drumlins can form very fast indeed. Calculations involving till sliding only and covering the same parameter range found no cases where the growth rate was positive. This suggests that drumlins which have formed from till slipping over its bed may also be erosional remnants or may arise from moulding of existent relief.

A fundamental constraint on any linearization technique is that it makes no prediction of what happens when the perturbation becomes large. The small parameter in the NK perturbation is the bed slope and the perturbation becomes invalid when the slope reaches unity. However, when the wavelength is $100 \mathrm{~m}$ and the perturbation amplitude $10 \mathrm{~m}$, the error in the Stokes equations is still only $10 \%$, meaning that qualitative predictions may remain correct provided that the original till thickness was sufficiently large that a $10 \mathrm{~m}$ amplitude wave remains small.

By the time slopes reach order unity, we anticipate shock formation to have occurred, which is not a process that can occur in the present linearized theory. A further factor likely to be of significance is the appearance of higher harmonics when the perturbation of a linear fluid is considered to higher order (Gudmundsson, 1997a) and (perhaps separately) when a fluid with a Glen rheology is considered (Gudmundsson, 1997b). These analyses show that higherorder and non-linear effects start to play a significant role well before the aspect ratio reaches order unity. These factors could cause relief to be amplified at different wavelengths from that of the perturbation.

\section{CONCLUSIONS}

This paper has been concerned with establishing a fundamental property of ice shearing over sediments deforming according to a double power-law rheology; does it act so as to amplify relief? The answer is that, under certain condi-
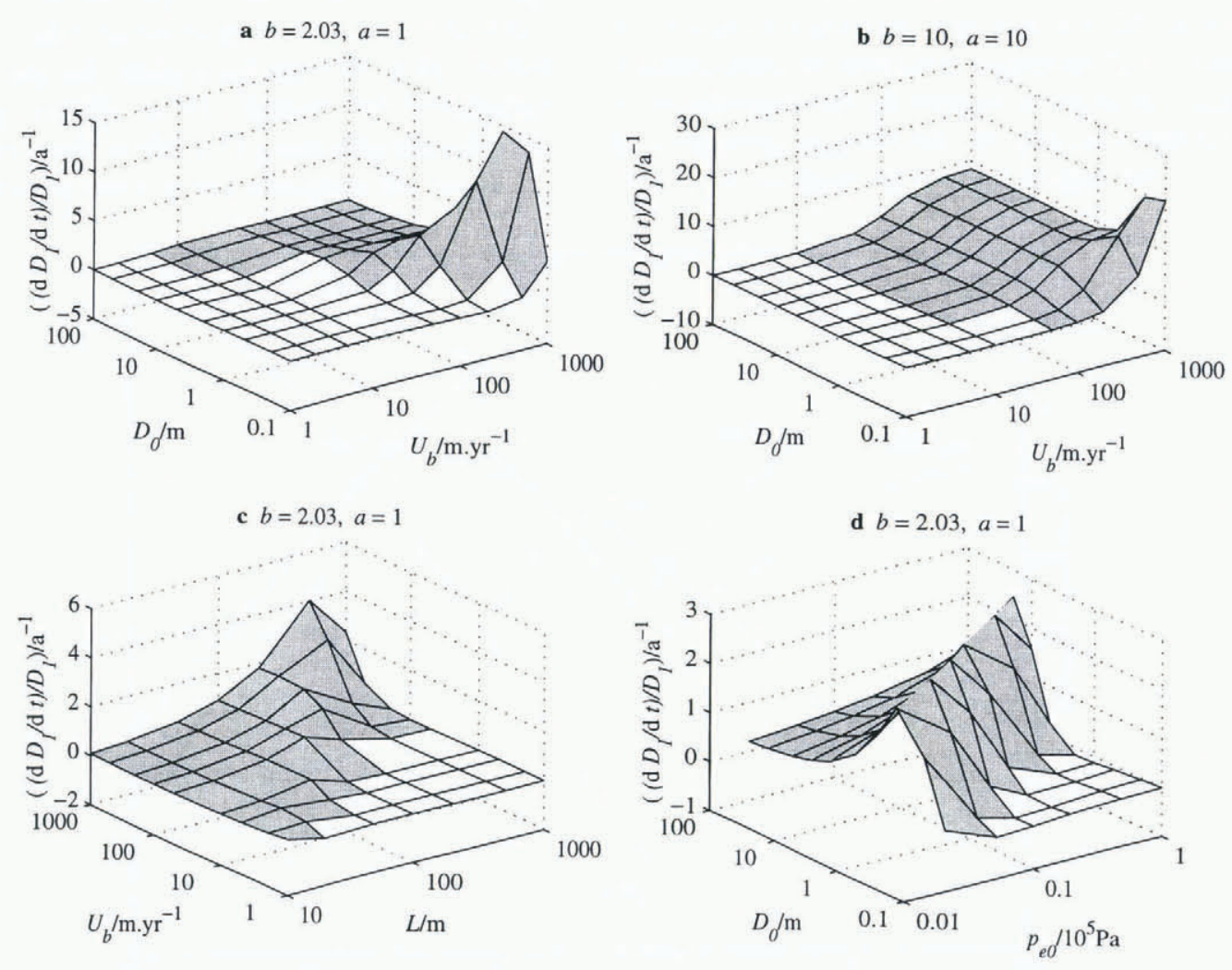

Fig. 2. Plots of growth-rate constant $\dot{D}_{\mathrm{o}}^{\mathrm{c}} / D_{\mathrm{o}}^{\mathrm{c}}$ as a function of indicated independent variables, and for various cases of the indices $a$ and $b$. In cases where they are not, the independent variables, $D_{0}=10 \mathrm{~m}, U_{\mathrm{b}}=100 \mathrm{ma}^{-1}, L=100 \mathrm{~m}, p_{\mathrm{e}}=10^{5} \mathrm{~Pa}$, and $\tau=10^{5} \mathrm{~Pa}$. Growth rates are shaded where positive and white where negative. 
tions, infinitesimal perturbations grow. How large they grow and, whether they will continue to grow once shocks start to form, is not answered by this analysis. This analysis is taken as indicating that drumlins could be formed from a till-sheet flow instability, and is consistent with a body of evidence (Boulton, 1987; Hart, 1997) that drumlins are sometimes composed of deformation till and are not necessarily formed by the moulding of pre-existent relief.

The drumlin instability therefore represents another aspect of larger-scale behaviour which can be modelled using viscous laws. Framed as they are at the larger scale, there is no reason why they should represent smaller-scale behaviour and it is a misconception to suppose that they must. Furthermore, the viscous-type law must, on the present state of knowledge, be more appropriate for large-scale icesheet modelling than the plastic law.

\section{ACKNOWLEDGEMENT}

I should like to thank A. Fowler for pointing out a mistake in another paper which led to the development of the theory in this paper.

\section{REFERENCES}

Alley, R. B. 1989. Water-pressure coupling of sliding and bed deformation: II. Velocity-depth profiles. 7. Glaciol., 35(119), 119-129.

Bahr, D. B. and J. B. Rundle. 1996. Stick-slip statistical mechanics at the bed of a glacier. Geophvs. Res. Lett. 23 16). 2073-2076.

Blankenship, D. D., C. R. Bentley, S. T. Rooney and R. B. Alley. 1986. Seismic measurements reveal a saturated porous laver beneath an active Antarctic ice stream. Nature, 322 (6074), 54-57.

Boulton, G. S. 1987. A theory of drumlin formation by subglacial sediment deformation. In Menzies, J. and J. Rose, eds. Drumlin Symposium. Rotterdam, A.A. Balkema, 25-80.

Boulton, G. S. and R. C. A. Hindmarsh. 1987. Sediment deformation beneath glaciers: rheology and geological consequences. F. Geophys. Res., 92 B 39,90599082

Fowler, A. C. 1981. A theoretical treatment of the sliding of glaciers in the absence of cavitation. Philos. Trans. R. Soc. London, Ser. A, 298 (1445), 637-685.

Gudmundsson, G. H. 1997a. Basal flow characteristics of a linear medium sliding frictionless over small bedrock undulations. F. Glaciol., 43 (143), 71-79.

Gudmundsson, G. H. 1997b. Basal flow characteristics of a non-linear flow sliding frictionless over strongly undulating bedrock. F. Glaciol., 43 (143), 80-89.

Hart, J. K. 1997. The relationship between drumlins and other forms of subglacial glaciotectonic deformation. Quat. Sci. Rev., 16, 93-107.

Hindmarsh, R. C. A. 1996. Sliding of till over bedrock: scratching, polishing, comminution and kinematic-wave theory. Ann. Glaciol, , 22, 41-47.

Hindmarsh, R. C. A. 1997. Deforming beds: viscous and plastic scales of deformation. Quat. Sci. Rev., 16, 1039-1056.

Hindmarsh, R. C. A. In press. Drumlinization and drumlin-forming instabilities: viscous till mechanisms. F. Glaciol.

Iverson, N. R., B. Hanson, R. LeB. Hooke and P. Jansson. 1995. Flow mechanism of glaciers on soft beds. Science, 267 (5194), 80-81.

Kamb, B. 1970. Sliding motion of glaciers: theory and observation. Rev. Geophys. Space Phys, 8 4), 673-728.

Kamb, B. 1991. Rheological nonlinearity and flow instability in the deforming bed mechanism of ice stream motion. J. Geophys. Res., 96 (Bl0), $16,585-16,595$.

Morland, L.W. 1976a. Glacier sliding down an inclined wavy bed. J. Glaciol., 17 (77), 447-462.

Morland, L. W. 1976b. Glacier sliding down an inclined wavy bed with friction. J. Glaciol., $17(77), 463-477$.

Nye, J. F. 1969. A calculation on the sliding of ice over a wavy surface using a Newtonian viscous approximation. Proc. R. Soc. London, Ser. A, 311 (1506), $445-467$.

Nye, J. F. 1970. Glacier sliding without cavitation in a linear viscous approximation. Proc. R. Soc. London, Ser. A, 315 1522, 381-403.

\section{APPENDIX}

\section{NYE TRANSFORMATION UNDER UNIFORM SHEAR}

Consider a region periodic in the horizontal direction in the domain $\tilde{x}=[0,2 \pi / k]$ and $\tilde{z} \geq 0$. Define horizontal and vertical velocities $\tilde{u}, \tilde{w}$ and pressure $\tilde{p}$. The Stokes equations

$$
\begin{aligned}
\eta\left(\frac{\partial^{2} \tilde{u}}{\partial \tilde{x}^{2}}+\frac{\partial^{2} \tilde{u}}{\partial \tilde{z}^{2}}\right) & =\frac{\partial p}{\partial \tilde{x}}, \\
\eta\left(\frac{\partial^{2} \tilde{w}}{\partial \tilde{x}^{2}}+c \frac{\partial^{2} \tilde{w}}{\partial \tilde{z}^{2}}\right) & =\frac{\partial p}{\partial \tilde{z}}, \\
\frac{\partial \tilde{u}}{\partial \tilde{x}}+\frac{\partial w}{\partial \tilde{z}} & =0
\end{aligned}
$$

where $\eta$ is the viscosity of ice approximated as a linear fluid. On the base $\tilde{z}=\tilde{D}(x)$, we have boundary conditions

$$
\begin{aligned}
\tilde{T}^{\mathrm{t}}= & \frac{\eta}{1+\left(\partial_{\tilde{x}} \tilde{D}\right)^{2}} \times \\
& \left(\left(\frac{\partial \tilde{u}}{\partial \tilde{z}}+\frac{\partial \tilde{w}}{\partial \tilde{x}}\right)\left(1-\left(\partial_{\tilde{x}} \tilde{D}\right)^{2}\right)+4 \partial_{\tilde{x}} \tilde{D} \frac{\partial \tilde{u}}{\partial \tilde{x}}\right), \\
\tilde{T}^{\mathrm{n}}=\tilde{p} & +\frac{2 \eta}{1+\left(\partial_{\tilde{x}} \tilde{D}\right)^{2}}\left(\frac{\partial \tilde{u}}{\partial \tilde{x}}\left(1-\left(\partial_{\tilde{x}} \tilde{D}\right)^{2}\right)\right. \\
& \left.+\left(\frac{\partial \tilde{u}}{\partial \tilde{z}}+\frac{\partial \tilde{w}}{\partial \tilde{x}}\right)\left(\partial_{\tilde{x}} \tilde{D}\right)\right) .
\end{aligned}
$$

For convenience, we have adopted the compressive-positive convention for the normal traction $\tilde{T}^{\mathrm{n}}$.

$\because$ can generate a zeroth-order solution by assuming that the bed is flat

$$
\tilde{D}=\tilde{D}_{0}
$$

and that a traction $T_{0}^{\mathrm{t}}$ is applied to a horizontal surface on the ice at a distance very far from the bed. This sets up a simple shear in the ice and also creates a horizontal velocity in the ice at the bed $z=0$. We find

$$
\tilde{u}_{0}=U_{\mathrm{b}}+\partial_{z} U \tilde{z}, \partial_{z} U=\tilde{T}_{0}^{\mathrm{t}} / \eta .
$$

Following Nye (1969, 1970) and Kamb (1970), we perturb the zeroth-order solution by introducing some undulations into the bed. There is a difference, because we have a base solution with simple shear. The perturbation expansion is

$$
\begin{aligned}
\tilde{u} & =U_{\mathrm{b}}+\partial_{z} U \tilde{z}+\varepsilon \tilde{u}_{1}(\tilde{x}, \tilde{z})+O\left(\varepsilon^{2}\right), \\
\tilde{w} & =\varepsilon \tilde{w}_{1}(\tilde{x}, \tilde{z})+O\left(\varepsilon^{2}\right), \tilde{p}=\varepsilon \tilde{p}_{1}(\tilde{x}, \tilde{z})+O\left(\varepsilon^{2}\right), \\
\tilde{D} & =\tilde{\varepsilon} \tilde{D}_{1}(\tilde{x})+O\left(\varepsilon^{2}\right), \\
\tilde{T}^{\mathrm{n}} & =\tilde{T}_{0}^{\mathrm{n}}+\varepsilon \tilde{T}_{1}^{\mathrm{n}}, \tilde{T}^{\mathrm{t}}=\tilde{T}_{0}^{\mathrm{t}}+\varepsilon \tilde{T}_{1}^{\mathrm{t}} .
\end{aligned}
$$

We now use the Nye transform to account for zeroth-order shear

$$
\begin{aligned}
& x=\tilde{x}, z=\tilde{z}-\varepsilon D, \\
& u=\tilde{u}, w=\tilde{w}, p=\tilde{p}
\end{aligned}
$$

and write down another set of perturbation equations where the zeroth-order solution is 


$$
\begin{aligned}
u & =U_{\mathrm{b}}+\partial_{z} U\left(z+\varepsilon D_{1}\right)+\varepsilon u_{1}(x, z)+O\left(\varepsilon^{2}\right), \\
w & =\varepsilon w_{1}(x, z)+O\left(\varepsilon^{2}\right), \quad p=\varepsilon p_{1}(x, z)+O\left(\varepsilon^{2}\right), \\
D & =\varepsilon D_{1}(x)+O\left(\varepsilon^{2}\right), \\
T^{\mathrm{n}} & =T_{0}^{\mathrm{n}}+\varepsilon T_{1}^{\mathrm{n}}, T^{\mathrm{t}}=T_{0}^{\mathrm{t}}+\varepsilon T_{1}^{\mathrm{t}} .
\end{aligned}
$$

It is straightforward to show that

$$
\begin{aligned}
& \tilde{u}(\tilde{x}, \tilde{z}=\zeta)-u(x, z=\zeta)= \\
& \varepsilon \partial_{z} U D_{1}+\varepsilon \tilde{u}_{1}(\tilde{x}, \tilde{z}=\zeta)-\varepsilon u_{1}(x, z=\zeta)+O\left(\varepsilon^{2}\right)
\end{aligned}
$$

which implies that one can write with error $O(\varepsilon)$ that

$$
\tilde{u}_{1}(\tilde{x}, \tilde{z}=\zeta)=u_{1}(x, z=\zeta)-\partial_{z} U D_{1}
$$

and one may also write to the same accuracy $\tilde{w}_{1}(\tilde{x}, \tilde{z}=\zeta)=w_{1}(x, z=\zeta), \tilde{p}_{1}(\tilde{x}, \tilde{z}=\zeta)=p_{1}(x, z=\zeta)$.

(This is what Nye (1970) did but we need to be more careful with our notation owing to the presence of shear in the zerothorder solution.)
We can readily obtain the differential transforms

$$
\begin{aligned}
\frac{\partial}{\partial \tilde{x}} & =\frac{\partial}{\partial x}-\varepsilon \partial D_{1} \partial x \frac{\partial}{\partial z}, \\
\frac{\partial^{2}}{\partial \tilde{x}^{2}} & =\frac{\partial^{2}}{\partial x^{2}}-\varepsilon\left(\frac{\partial^{2} D_{1}}{\partial^{2} x} \frac{\partial}{\partial z}+\frac{\partial D_{1}}{\partial x} \frac{\partial^{2}}{\partial z \partial x}\right)+\varepsilon^{2} \frac{\partial D_{1}}{\partial x} \frac{\partial^{2}}{\partial z^{2}}
\end{aligned}
$$

and it is then easy to show that we obtain the following perturbation equations

$$
\begin{aligned}
\eta \nabla^{2} u_{1} & =\partial_{x} p_{1}+\partial_{z} U \partial_{x}^{2} D_{1}, \\
\eta \nabla^{2} w_{1} & =\partial_{z} p_{1}, \\
\partial_{x} u_{1}+\partial_{z} w_{1} & =\partial_{z} U \partial_{x} D_{1},
\end{aligned}
$$

with boundary relations

$$
\begin{aligned}
T_{1}^{\mathrm{t}} & =\eta\left(\frac{\partial u_{1}}{\partial z}+\frac{\partial w_{1}}{\partial x}\right), \\
T_{1}^{\mathrm{n}} & =-\sigma_{z z_{1}}=\tau_{x x_{1}}+p_{1}=2 \eta\left(\frac{\partial u_{1}}{\partial x}-\partial_{z} U \partial_{x} D_{1}\right)+p_{1} .
\end{aligned}
$$

